Funding: [See page 104]

Peer review: This article has been subject to a double blind peer review process

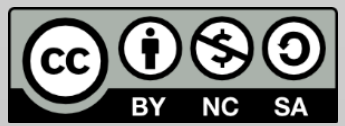

(C) Copyright: The Authors. This article is issued under the terms of the Creative Commons Attribution NonCommercial Share Alike License, which permits use and redistribution of the work provided that the original author and source are credited, the work is not used for commercial purposes and that any derivative works are made available under the same license terms.

\section{Modelling and Simulations of a Narrow Track Tilting Vehicle}

\section{J. J. Chong*, James Marco, David Greenwood}

WMG, University of Warwick, Coventry, CV4 7AL, UK

*Correspondence: j.chong@warwick.ac.uk

\begin{abstract}
Narrow track tilting vehicles are a new category of vehicles that combine the dynamical abilities of a passenger car with a motorcycle. In the presence of unstable moments during cornering, an accurate assessment of the lateral dynamics plays an important role to improve the stability and handling of the vehicle. In order to stabilise or control the narrow tilting vehicle, the demand tilt angle can be calculated from the vehicle's lateral acceleration and controlled by either steering input of the vehicle or using additional titling actuator to reach this desired angle. The aim of this article is to present a new approach for developing the lateral dynamics model of a narrow track tilting vehicle. First, this approach utilises the well-known geometry 'bicycle model' and parameter estimation methods. Second, by using a tuning method, the unknown and uncertainties are taken into account and regulated through an optimisation procedure to minimise the model biases in order to improve the modelling accuracy. Therefore, the optimised model can be used as a platform to develop the vehicle control strategy. Numerical simulations have been performed in a comparison with the experimental data to validate the model accuracy.
\end{abstract}

Keywords: narrow track vehicle, modelling, titling control, vehicle lateral dynamics, parameter estimation

\section{Introduction}

European Union (EU) cities are increasingly congested due to the demand and usage of motor vehicles that results in increased emissions, increased noise levels and scarcer parking, which affects the quality of life and health of city-dwellers. To address such issues, European-wide emission targets are becoming stricter and urban mobility plans are being drawn up. 
It is expected that narrow track vehicles (NTVs) would be the next generation of mobility. They are the convergence of a car and a motorcycle, i.e. with smaller dimensions and reduced energy consumption and pollutant emission. However, the narrow track of the vehicle itself increases the tendency to become unstable during cornering when facing lateral acceleration. This problem can be mitigated by tilting the vehicle in a way that reduces the lateral acceleration of the vehicle during cornering, and would self-stabilise the vehicle similarly to a motorcycle. So far in the existing literature, the corresponding tilting angle is computed and the control strategy aims at reaching this desired angle.

In order to facilitate the tilting control on a NTV, it is important to have a plant model that can emulate the vehicle accurately and to be executed in real-time without any loss of simulation accuracy or numerical stability. Generally, the modelling approach for vehicle dynamic analysis can be divided into two categories; the multi-body model and the simplified model. The multi-body model can accurately represent the kinematic and compliance characteristics of the vehicle. However, the heavy computational process with the details of the chassis is not suitable for a real-time hardware-in-the-loop simulation (HILS) system. In contrast, the simplified model of the vehicle is a rather simple model structure that is frequently being used in the vehicle dynamics literature and is more suitable for real-time simulation.

An initial analysis of the narrow track tilting vehicle has been published by Kidane et al., 2008. , to explore the research of this new category of vehicle. A large part of the work has been focused on the modelling of titling vehicles in order to investigate the strategies employed to control the stability of the vehicle by Gohl et al., 2004. Furuichi et al., 2012 derive a new switching dynamical model of NTV. This model considers several NTV states including normal running, temporary running with one rear wheel not on the ground and totally falling down.

A multibody Adams/Motorcyle model has been developed by Amati et al., 2011, and cross-validated by the SimMechanics (Besselink, 2006) model to study the lateral vibration motions of the tilting vehicle. A further complex system has been developed by Maakaroun et al., 2011, using a systematic model design from robotics techniques. This approach automatically calculates the symbolic expression of the geometric, kinematic and dynamic models by using a symbolic software package SYMORO+. In addition, The Compact Low Emission Vehicle for Urban Transport (CLEVER) prototype (Berote, 2010) was developed as part of an EU funded consortium with extensive modelling and control works carried out by the University of Bath. 
The parameters of the models are one of the important factors that determine the accuracy of the system modelling and eventually the overall performance of the closed-loop system. The parameter estimates may be used directly in a control or state-estimation algorithm - such algorithms are often referred to as adaptive algorithms. For example, Ryu (2004) carried out his research at Stanford University to estimate the vehicle parameters of tyre stiffness; yaw moment of inertia; and roll stiffness using the least squares method and total least squares method.

Several studies have already been made on similar topics (Gerdes et al., 2006; Lundquist, 2009).These papers use a well-known single track model and tyre force model to estimate the road-tyre interaction parameters such as side slip angle and tyre force. All these papers use identification methods to evaluate the unknowns from actual vehicle sensors.

The objective of this article is to develop a dynamic model for NTVs that can be used for the evaluation of the tilting control system. The modelling approach uses a geometric 'bicycle' model and parameter estimation methods to represent the lateral dynamics of the vehicle for better representation of kinematic and compliance characteristics of the tyres. The validity of the proposed method is demonstrated by comparing the simulation results of the lateral acceleration model with field test results.

\section{Narrow Tilting Vehicle Dynamics and Control Strategies}

\subsection{Tilting control strategies}

Based on the aforementioned tilting control strategies of NTVs, the roll acceleration was assumed to balance the components of the lateral acceleration and the gravitational acceleration of the tilt assembly. The control strategy utilises the vehicle longitudinal speed and front wheel steer angle to predict the lateral acceleration and hence the tilting angle required to balance the vehicle during cornering can be described as:

$$
\varphi_{\text {desired }}=\frac{\boldsymbol{a}_{y}}{\boldsymbol{g}}
$$

where $\varphi_{\text {desired }}$ denotes the desired tilting angle; $\boldsymbol{a}_{y}$ is the lateral acceleration of the vehicle; and $\boldsymbol{g}$ is the acceleration due to gravity.

The following section describes two common tilting systems i.e., direct tilt control (DTC) and steering tilt control (STC), that take the lateral 
acceleration as reference, to control the desired tilt angle. Error! Reference source not found.

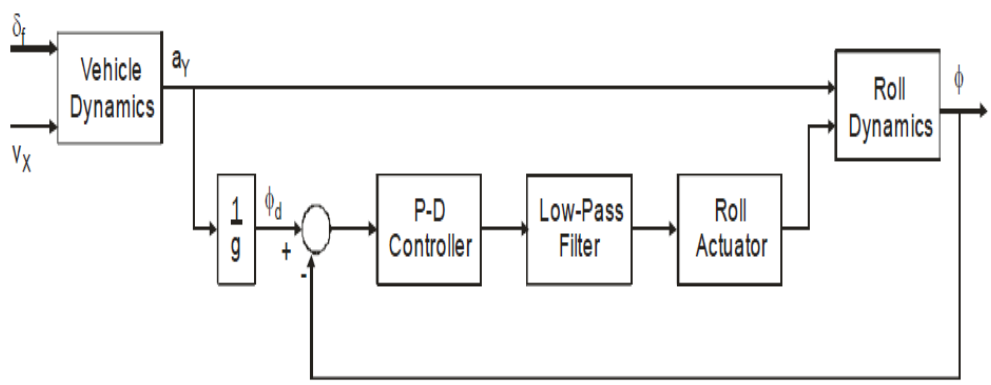

(a)

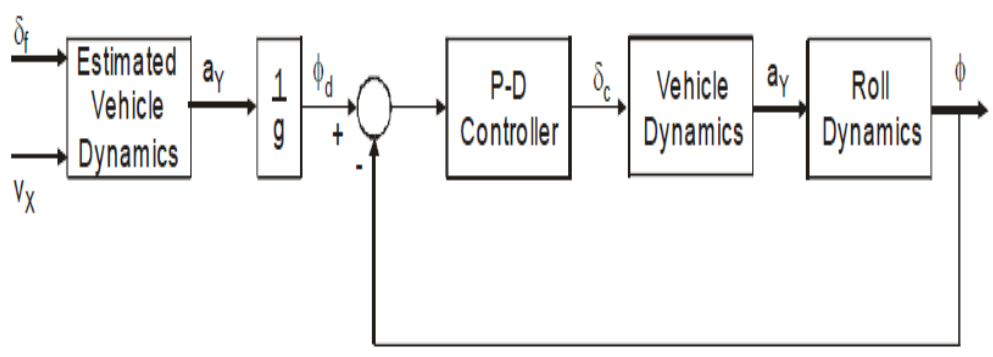

(b)

Fig. 1. Tilting Control Strategies: (a) Direct Tilt Control (left); (b) Steer Tilt Control (right)

describes two common tilting systems i.e., DTC and STC, systems proposed by So et al., 1997. As can be seen in the figures 1 (a) and (b) to implement the concept of tilting control, first, a geometric vehicle model is necessary to calculate the lateral acceleration of the vehicle, and secondly, a concept map is required to describe the structure of the tilting.

Both the control strategies take the steer angle at the front wheel $\left(\delta_{f}\right)$ and the longitudinal vehicle velocity $\left(V_{x}\right)$ as the inputs to the system. In the DTC, a Proportional-Derivative (PD) controller and a roll actuator have been implement to control the titling error that compared the demanded tilt angle and the actual tilt angle. The output of the control scheme is a moment that is applied to the tilting body. In contrast, the STC utilised the PD controller with negative gain, tilting the vehicle in opposite direction to the later force, for example to tilt the vehicle to the right, a lateral force is required to balance on the left and vice versa. This steer angle is the angle at which the vehicle will be steered instead of the driver steering input. In other words, the control steer is applied instantaneously and the steer angle immediately generates a lateral acceleration, this control phenomenon can also be called counter steering. 


\subsection{Roll model}

To analyse the tilting control systems a roll degree of freedom is added. One of the most common methods is the inverted pendulum model (Error! Reference source not found.). The principle of the tilting control of the tiling vehicle has some characteristics such as instability, multivariablity and non-linearity, similar to the inverted pendulum system to balance the pendulum and control the lateral motion of the cart. The roll equation for an inverted pendulum combined with the definition of the lateral acceleration can be described in the following:

$$
\ddot{\emptyset}_{\text {pendulum }}=\frac{m g h_{c} \sin \emptyset-m h_{c}\left(a_{y}\right) \cos \emptyset+M_{\text {tilt }}}{\left(m h_{c}{ }^{2}+I_{x x}\right)}
$$

where $M_{\text {tilt }}$ denotes the tilting torque delivered by the dedicated actuator; $I_{X x}$ is the inertia moment of the X-axis; $h_{c}(\mathrm{~m})$ is the height of the centre gravity relative to the road surface, $m(\mathrm{~kg})$ is the mass of the vehicle; $g\left(\mathrm{~m} / \mathrm{s}^{2}\right)$ is the gravitational acceleration; and $\phi(\mathrm{rad})$ is the roll angle

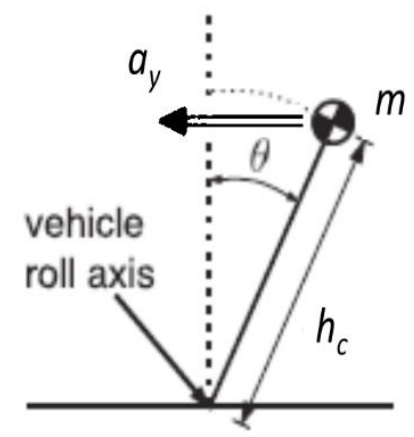

Fig. 2. Vehicle roll axis at ground level

As only the lateral motion of the vehicle is being considered, the effects of fore and aft load transfer resulting from braking, accelerating and air drag have been omitted.

\subsection{Lateral acceleration using a geometric 'bicycle' model}


Error! Reference source not found. shows a schematic of the geometric 'bicycle' model. The vehicle lateral motion can be developed by a mathematical description of the vehicle motion without considering the forces that affect the motion. This basic vehicle model is generally based on geometric relationships governing the kinematic of the vehicle system. Commonly the geometric steer angle is given in equation (3).

$$
\delta=\frac{l_{r}+l_{f}}{R}
$$

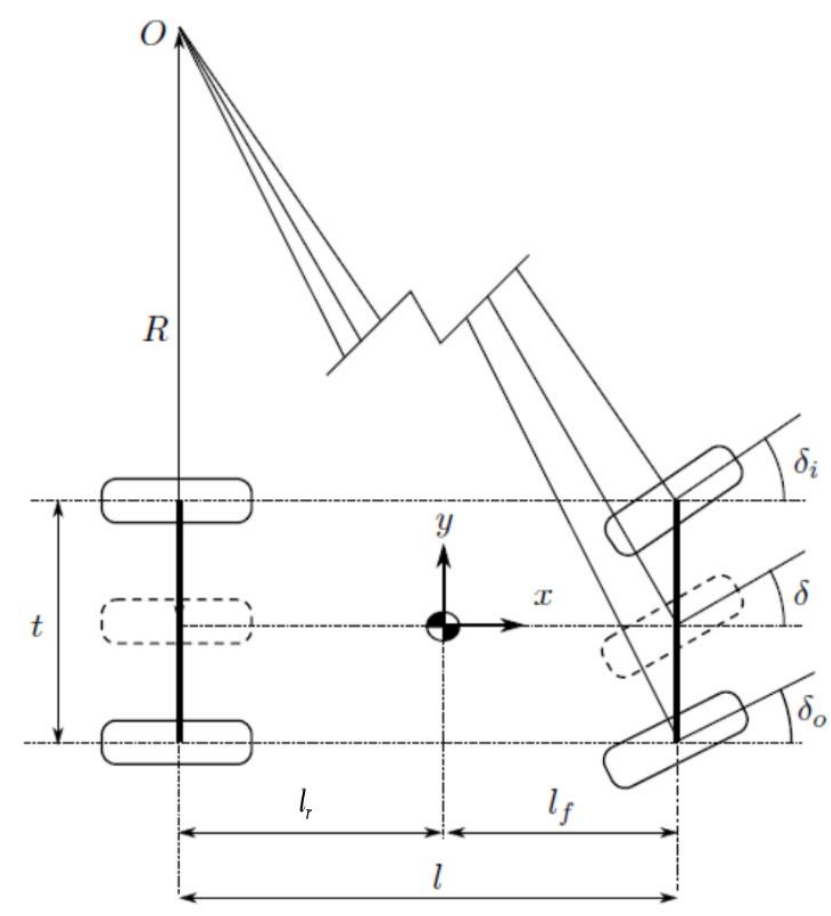

Fig. 3. Geometry of a turning vehicle (source: Griffin 2015)

When a vehicle turns at very low speed, its wheels can be assumed to roll perfectly, without slip, along the surface of the road. It is assumed that the lateral velocity is proportional to the velocity vector of the front wheel(s):

$$
v_{y}=\left(\frac{v_{x}}{l_{r}+l_{f}} l_{r} \delta\right)
$$

where $v_{x}$ and $v_{y}(\mathrm{~m} / \mathrm{s})$ is the vehicle longitudinal and lateral speed, respectively; $\delta(\mathrm{rad})$ is the front wheel steer angle, and $l_{f}$ and $I_{r}(\mathrm{~m})$ are the distance of front axle and real axle from COG $(\mathrm{m})$. 
It is also assumed radius of the vehicle path changes slowly due to low vehicle speed, then the yaw rate must be equal to the angular velocity of the vehicle.

$$
r=\frac{v_{x}}{R}
$$

where $r(\mathrm{rad} / \mathrm{s})$ is the yaw rate and $R(\mathrm{~m})$ is the turning radius;

The lateral acceleration $\left(a_{y}\right)$ consists of the rate of change in the lateral velocity $\left(\dot{v}_{y}\right)$ and the centripetal acceleration as shown in equation (6).

$$
a_{y}=\dot{v}_{y}+v_{x} r
$$

The rate change of the lateral velocity is defined by

$$
\dot{v}_{y}=\frac{1}{m}\left(F_{y_{-} t y r e(f)} \cos \delta+F_{y_{-} t y r e(r)}\right)-v_{x} r .
$$

where $F_{y_{-} t y r e}(\mathrm{~N})$ is the lateral tyre force, the subscript $(f)$ and $(r)$ is used to denote the front and rear respectively.

Combining equations (3), (4) and (5) into equation (6), the lateral acceleration is defined by equation (8).

$$
a_{y}=\frac{\dot{v}_{x}}{l_{r}+l_{f}} l_{r} \delta+\frac{v_{x}}{l_{r}+l_{f}} l_{r} \dot{\delta}+\frac{v_{x}^{2}}{l_{r}+l_{f}} \delta .
$$

The geometric bicycle model is very basic and relies on many assumptions (e.g. low vehicle speed and tyre slip angle being negligible). The dynamics of the model are dependent on the longitudinal velocity and the steering angle only and not on road-tyre interaction.

\subsection{Lateral acceleration using 'Bicycle' model and linear tyre model}




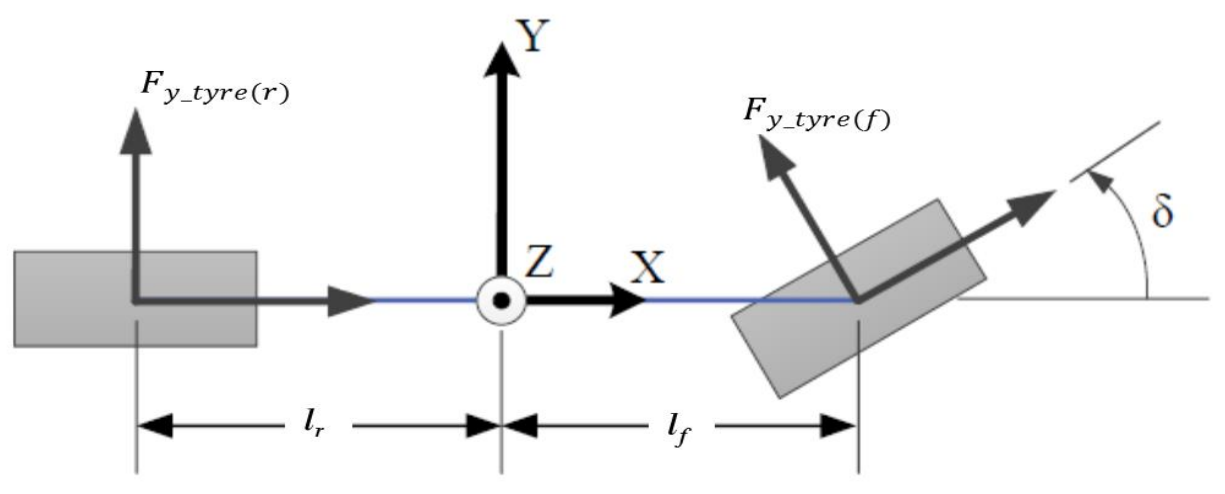

Fig. 4. Velocity and moments acting on the 'Bicycle' model

The geometric bicycle model is only valid for low speed cornering which follows the Ackermann steering geometry. To investigate high-speed cornering, a more complex description of the vehicle motion is necessary, and uses a simple vehicle description, known as 'single track model' or 'bicycle model'.

The lateral and yaw dynamics of a narrow tilting vehicle can be studied with the aid of a simple bicycle model shown in Fig. 4. Lateral motions are the result of force and moment generation through the road-tyre interaction. In conventional vehicle models, the lateral force is generated only by the steering angle, which is the resulting difference between the heading angle of the wheel and the wheel's velocity vector, called the side slip angle.

On the contrary, two wheeled vehicles (i.e. bikes and motorcycles) take benefit from the tilting angle to compensate for the fictitious inertial force effects when cornering, improving the stability and performance. The lateral force is not only generated by the side slip angle, but is also induced by the camber effect (Lu et. al, 2006). The latter outcome is generated by the dynamic change of the camber angle to ensure maximum contact of the tyre with the road surface during cornering.

Therefore for simplicity, the product of inertia and the gyroscopic influence by the tyres is neglected, the final linear model can be expressed in the following.

The lateral acceleration can be described by:

$$
a_{y}=\frac{1}{m}\left(F_{y_{-} t y r e(f)}+F_{y_{-} t y r e(r)}\right)
$$


where $m(\mathrm{~kg})$ is the mass of the vehicle, $F_{y_{-}}$tyre $(\mathrm{N})$ is the lateral tyre force, the subscript $(f)$ and $(r)$ is used to denote the front and rear, respectively

The yaw rate can be described by:

$$
\dot{r}=\frac{1}{I_{z z}}\left(l_{f} F_{y_{-} t y r e(f)}-l_{r} F_{y_{-} t y r e(r)}\right) .
$$

where $\mathrm{Izz}\left(\mathrm{kgm}^{2}\right)$ is the yaw inertia of the vehicle.

A steering input will generate a cornering force that makes a vehicle able to turn into a corner, which is known as a tyre side slip angle $(\alpha)$ :

$$
\begin{gathered}
\alpha_{f}=\delta-\tan ^{-1}\left(\frac{V_{y}+r l_{f}}{V_{x}}\right), \\
\alpha_{r}=-\tan ^{-1}\left(\frac{V_{y}-r l_{r}}{V_{x}}\right) .
\end{gathered}
$$

In the linear region of the tyre curve (small slip angle), the lateral force of the tyre can be described as:

$$
\begin{aligned}
& F_{y_{-} t y r e(f)}=C_{f} \alpha_{f}+C_{\gamma f} \gamma_{f}, \\
& F_{y_{-} \text {tyre }(r)}=C_{r} \alpha_{r}+C_{\gamma r} \gamma_{r} .
\end{aligned}
$$

A camber effect term is also included in the final expressions for the lateral tyre forces. The camber angle $\nu_{f}$ and $\nu_{r}$ are the proportionality gains from the lean angle. Where $C_{f}$ and $C_{r}$ is the front and rear tyre cornering stiffness coefficient $(\mathrm{Nm} / \mathrm{rad})$, and $C_{\gamma f}$ and $C_{\gamma f}$ is the front and rear camber coefficient $(\mathrm{Nm} / \mathrm{rad})$, respectively.

\subsection{Lateral acceleration using 'Bicycle' model and non-linear tyre model}

In real world applications, the tyre may work on the nonlinear region, for example, in a low friction surface. To describe this behaviour it would be necessary to make a new non-linear model. The non-linear force 
description of the motorcycle tyre is a set of equations that relate tyre load; lateral and longitudinal slips; and camber angle to lateral and longitudinal forces. The definitive form of the Magic Formula tyre model (Error! Reference source not found.) was presented by Pacejka et al., 1992.

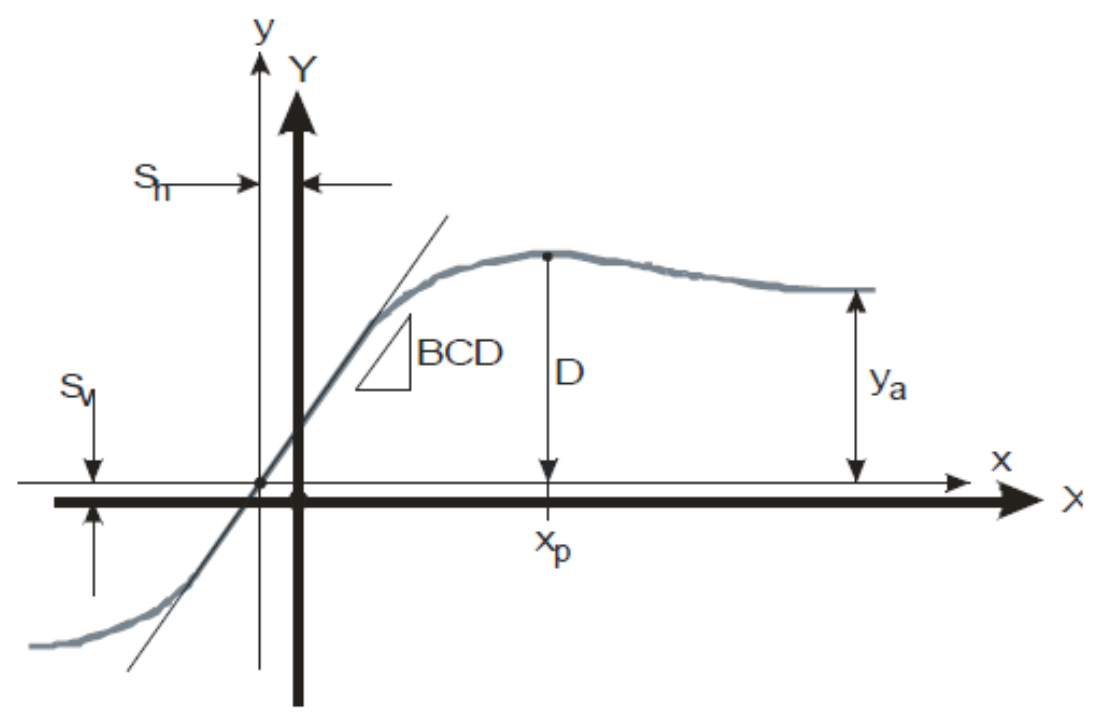

Fig. 5. Lateral tyre slip angle vs. lateral tyre force using Magic formula

The general form of the non-linear force description of the motorcycle tyre makes use of a simplified version of the magic formula for motorcycle tyres (Pacejka et al., 1992) can be described as:

$$
F_{y}(x)=D \sin \left[C \tan ^{-1}\left(B x-E\left(B x-\tan ^{-1}(B x)\right)\right)\right]
$$

where $x$ is the lateral tyre slip angle and $B, C, D$ and $E$ are the required model coefficients representing the_stiffness, shape factor, peak lateral force and curvature factor, respectively.

\subsection{Lateral acceleration using Geometric 'Bicycle' Model with parameter estimation}

Different approaches for estimating the lateral acceleration of a vehicle have been discussed in the previous section. The first two methods are not practical for physical world applications, as they are found to be simple and heavily reliant on the assumptions that hide the dynamic effects. In addition, the latter method of implementing the non-linear tyre model in the 'bicycle' model has many shortcomings, such as the 
model complexity; difficulty in measuring the slip change rate; and poor results when extrapolating outside the fitted range.

In order to overcome the limitations of these models, a modified geometric 'bicycle' model is proposed in which the model uncertainties are integrated into the geometric 'bicycle' model using an uncertain factor, $\sigma$. By neglecting the higher-order terms in equation (8), with an addition term $\sigma$ adding to equation (8), this equation can be re-written in the form:

$$
a_{y}=\frac{v_{x}^{2}}{l_{r}+l_{f}} \delta_{f}+\sigma
$$

were $\sigma$ is designated as uncertain parameter which is the function of vehicle longitudinal speed and steering wheel angle. It should be noted that, the $\sigma$ in equation (16) can be treated as constant parameter if the lateral acceleration of the vehicle is below $4 \mathrm{~m} / \mathrm{s}^{2}$ (Akar et al., 2008); or it can also be a time varying parameter if the vehicle is in a non-linear manoeuvres under the critical driving conditions. To simplify the model development, it is assumed that the $\sigma$ is a constant parameter and the vehicle is operated in a linear range.

Based on the experimental data and the proposed model, a least squares estimation of the uncertain parameter can be performed. The complete estimation algorithm has the following functionalities:

\section{Estimation algorithm}

\section{1: Initialise}

Set the $\sigma_{\text {in }}$ value to 0.01

\section{2: Calculate lateral acceleration, $a_{y}$}

With both the vehicle speed $\left(V_{x}\right)$ and steering angle $(\boldsymbol{\delta})$ known, the calculation of the lateral acceleration is based on Eq. 16. It can be rewritten as

$$
a_{y, \text { model }}=\frac{v_{x}^{2}}{l_{r}+l_{f}} \delta+\sigma_{i n}
$$




\section{3: Select training dataset to estimate $\sigma$ value}

To ensure that there is enough data for the parameter estimation process to be meaningful, maximum steering manoeuvre and maximum forward vehicle speed profile were selected as the training dataset in this study.

\section{4: Update $\sigma$ value}

This gives the starting point of the optimisation routine. The least square method defines the estimate of $\sigma$ value which minimises the sum of squares between the experimental acceleration and the lateral acceleration obtained from the proposed model. The optimisation sequence continues until a minimum error is found.

$$
\varepsilon=\sum_{n=1}^{N}\left(a_{y, \text { experimental }}-a_{y, \text { model }}\right)^{2}
$$

Where $\varepsilon$ represents the quality function, $\boldsymbol{N}$ is the total amount of samples in the training dataset, and $\boldsymbol{n}$ is the current sample.

$a_{y, \text { experimental }}$ and $a_{y \text {, model }}$ define as the lateral acceleration received from the experimental and from the proposed model, respectively.

\section{Simulation Results and Discussion}

In order to validate the model, steady state test results on a commercialised tilting vehicle (Honda Gyro, Fig. 6) from Poelgeest's PhD thesis (Poelgeest, 2011) is used to verified the concept of the lateral acceleration estimation using the proposed method.

Table 1 presents a representative dataset for the vehicle that can be employed to parameterise the model. For this test, as shown in Fig. 7, (black line illustrating the course, with a constant radius of $22 \mathrm{~m}$ ), small path deviations (light grey) were recorded during the vehicle driven on the circular path. During the test, the vehicle lateral acceleration, longitudinal speed and steering angle were synchronously recorded for the model optimisation and validation. 

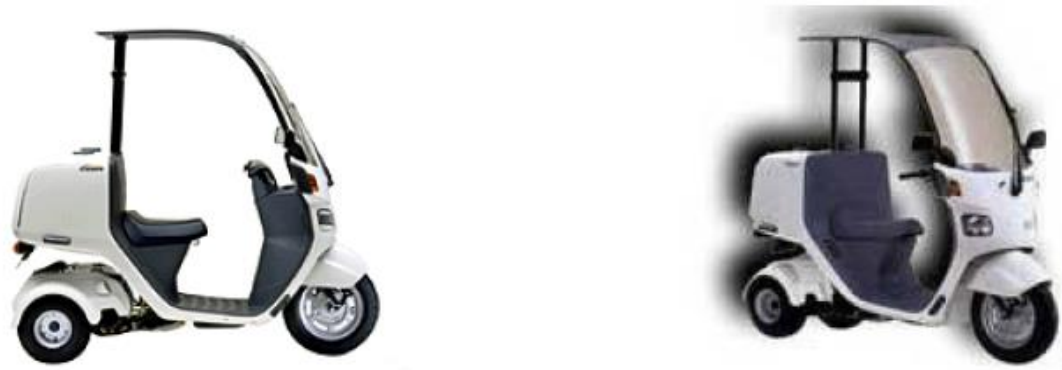

Fig. 6. Honda Gyro prototype - test vehicle (source: Poelgeest, 2011)

Table 1. Dataset for Honda Gyro Prototype

\begin{tabular}{ll}
\hline \hline Length & $1.895 \mathrm{~m}$ \\
\hline Width & $0.650 \mathrm{~m}$ \\
\hline Height & $1.690 \mathrm{~m}$ \\
Wheel base & $1.4 \mathrm{~m}$ \\
Weight & $136 \mathrm{~kg}$ \\
\hline
\end{tabular}

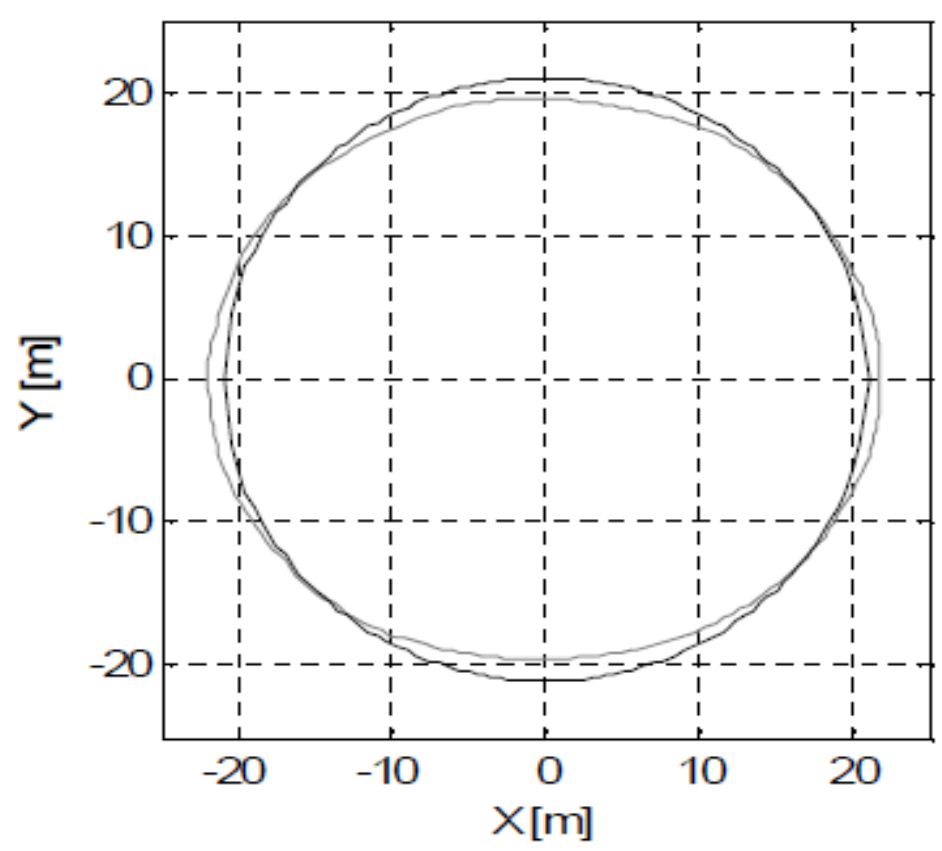

Fig. 7. 22m radius circle test (Poelgeest, 2011) 
As a result, the vehicle steering angle and longitudinal speed were obtained as plotted in Fig. 8 and

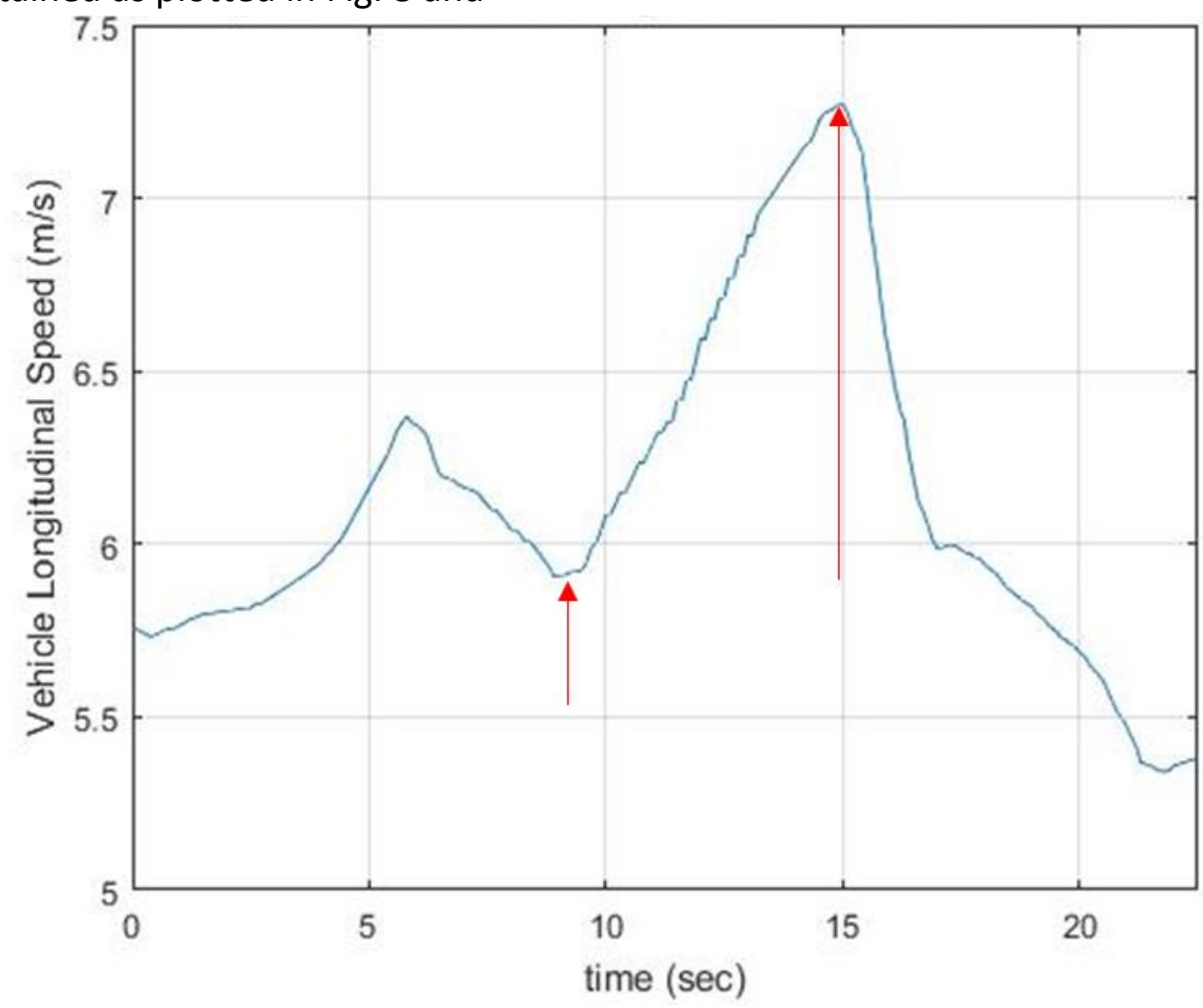

Fig. 9 and then used as the inputs for the model.

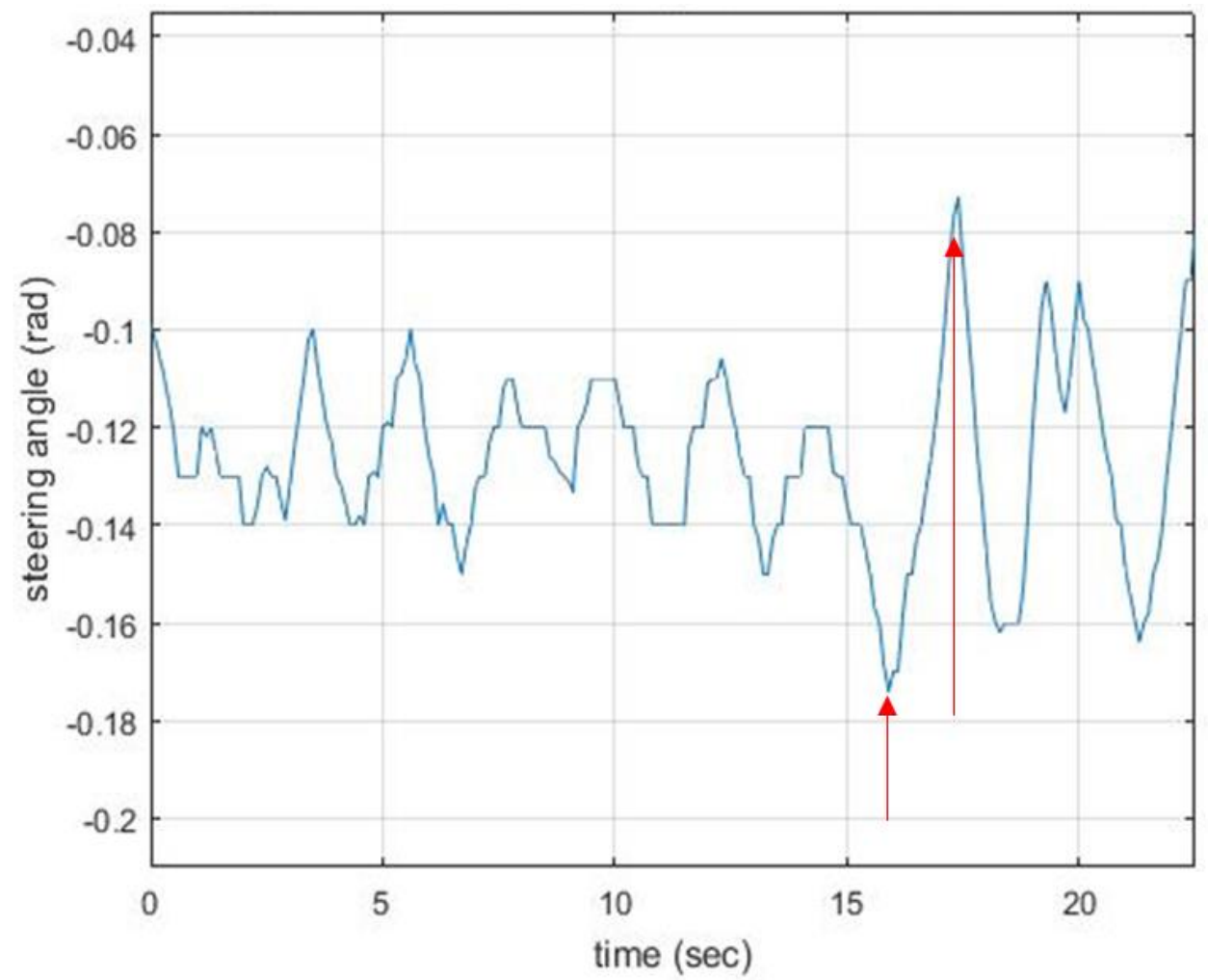


Fig. 8. Steer angle vs. time for one single turn around the circular track

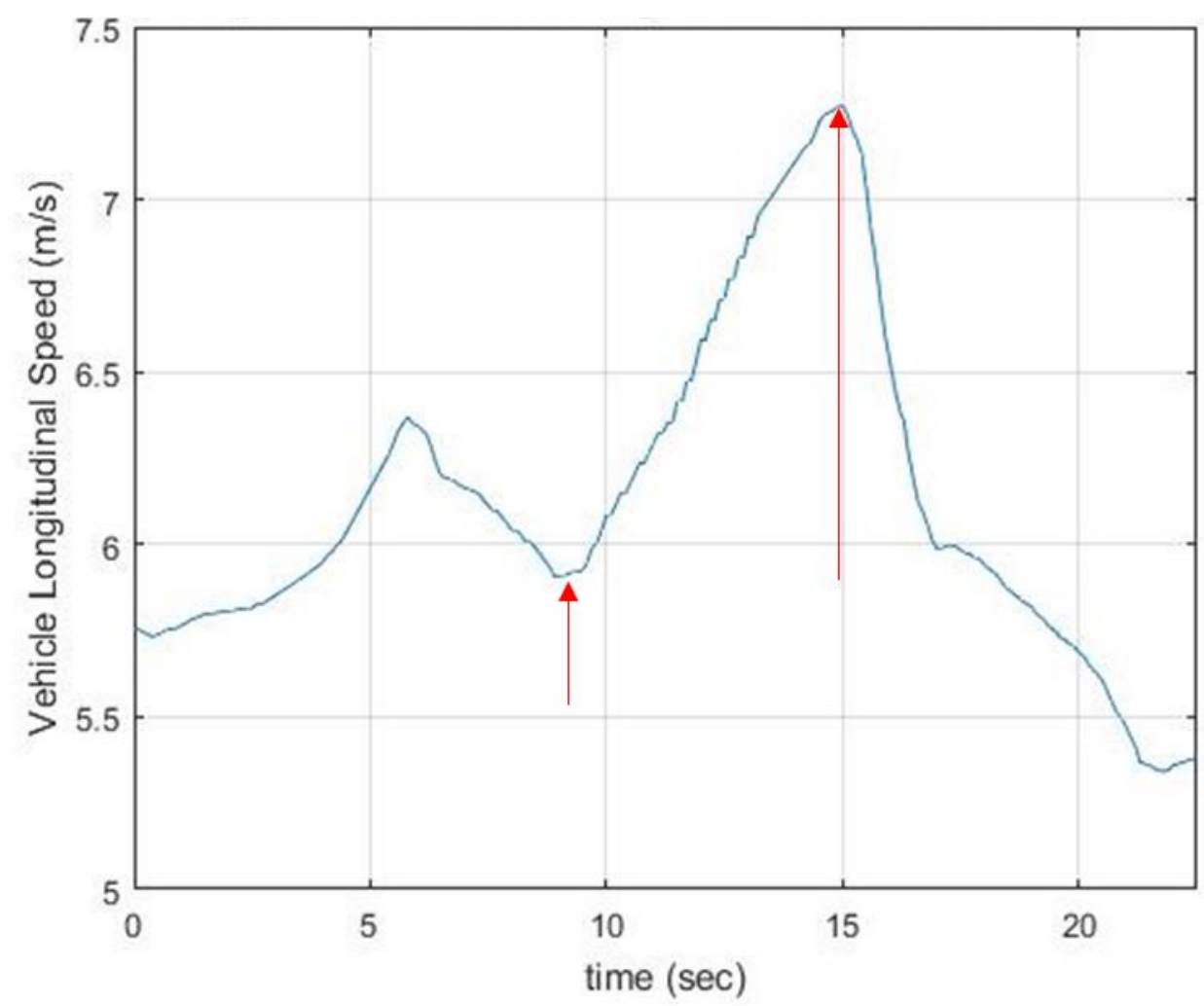

Fig. 9. Longitudinal speed vs. time for one single turn around the circular track

In order to identify the $\sigma$ factor in (16), the parameter estimation (as mentioned in Section 2.6) was performed in which a part of the data observation was selected for the training data set. Data ranges between 
the red arrows pointing in Fig. 8 and

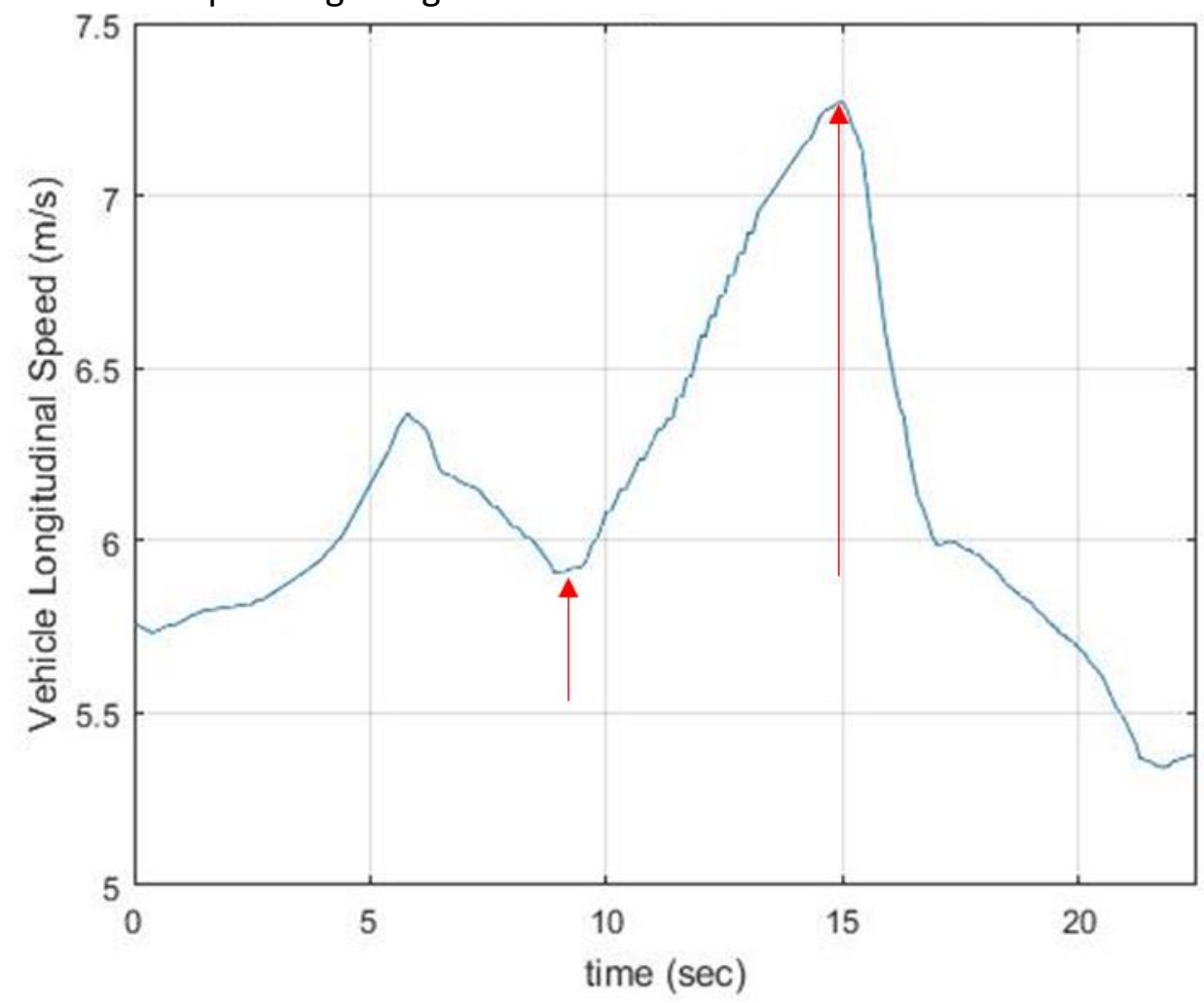

Fig. 9 indicate the chosen input training data. And the remained data were used for the model verification.

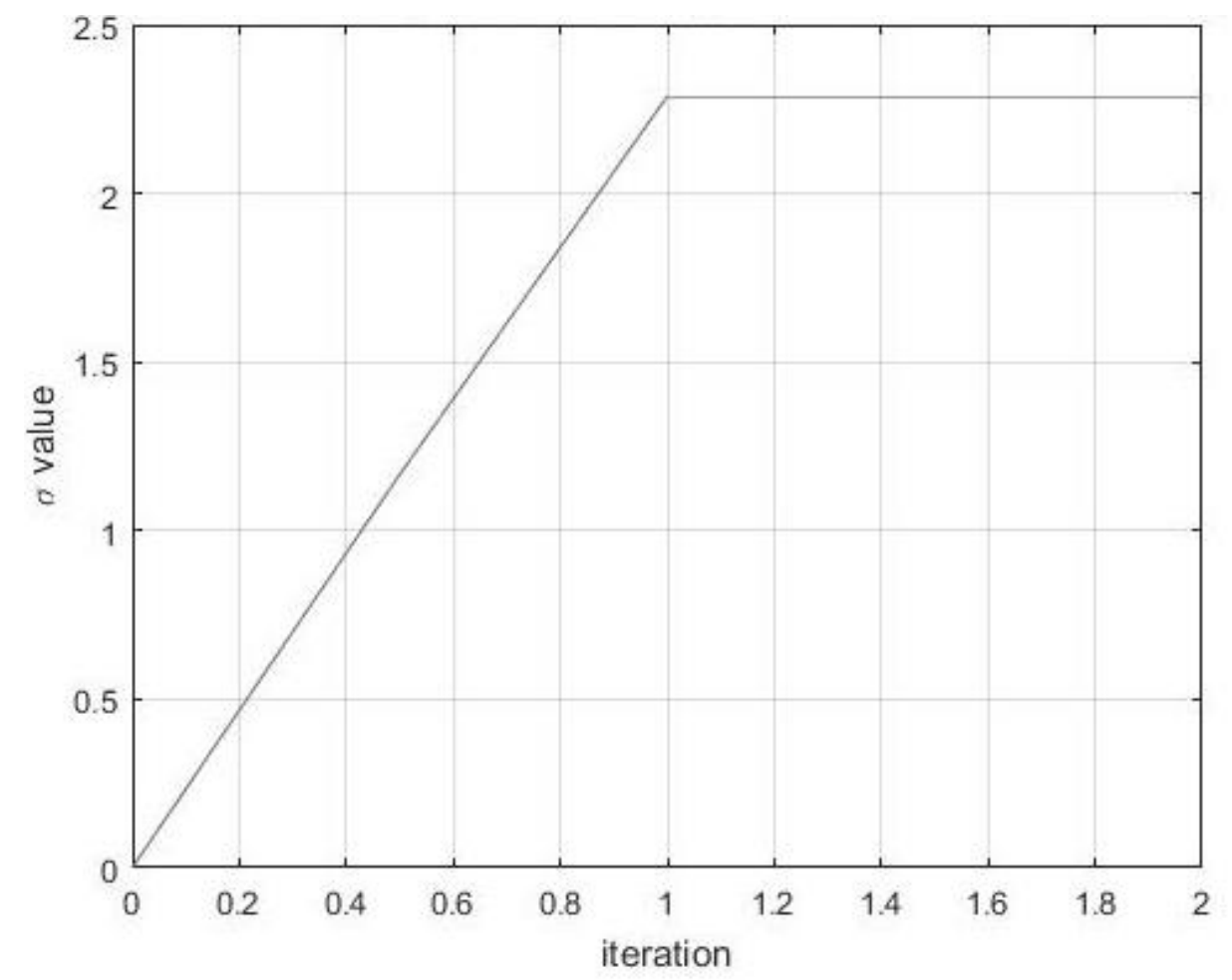


Fig. 10. Estimation of $\sigma$ value, identification by maximum forward vehicle speed

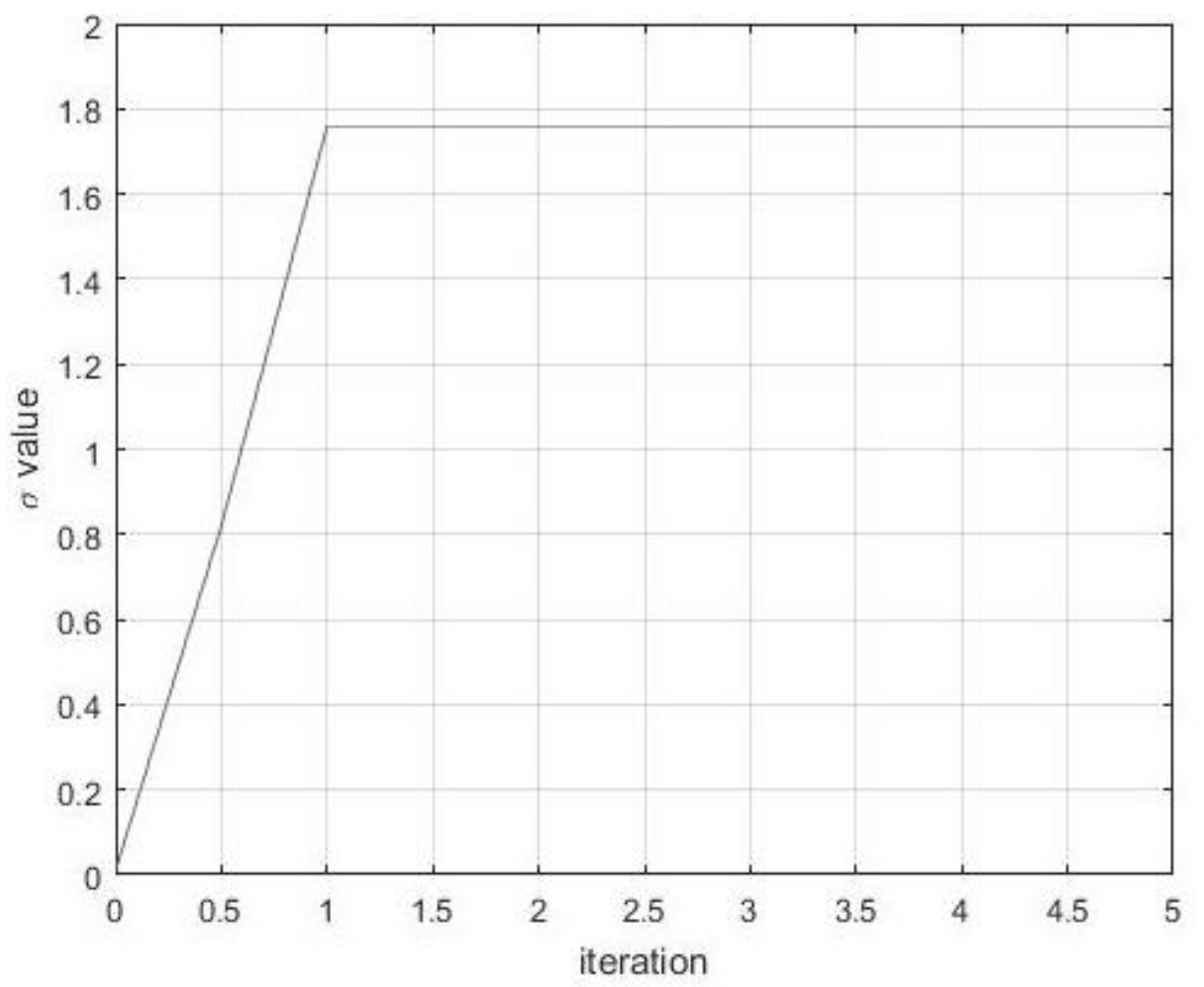

Fig. 11. Estimation of $\sigma$ value, identification by maximum steering manoeuvre

Fig. 10 and Fig. 11 show the estimation results of $\sigma$ from an experimental test by the least square method. To start the identification process, an initial value of 0.01 was given for the design variable. As can been noticed, the final values of 2.28 and 1.75 have been derived based on the selected data (according to the maximum forward vehicle speed and maximum steering manoeuvre), respectively. The estimates converged to the given values after less than five iterations. Next, these estimated parameters were implemented into the geometric 'bicycle' model as the constant parameter.

Finally, the optimal model was then tested with the experimental data to evaluate the modelling capability. Fig. 12 depicts the comparison between the actual lateral acceleration and the estimated one using the geometric 'bicycle' model with/without using the variable $\sigma$. It is obvious that the model without the adaptive factor, $\sigma$, was unable to represent the vehicle lateral acceleration accurately. Meanwhile, by using the proposed method in (16), derived from the parameter identification process, the model could estimate the vehicle lateral acceleration with an acceptable error under the tested conditions. This result confirms 
convincingly that the suggested approach can be used to simulate the lateral acceleration of the vehicle.

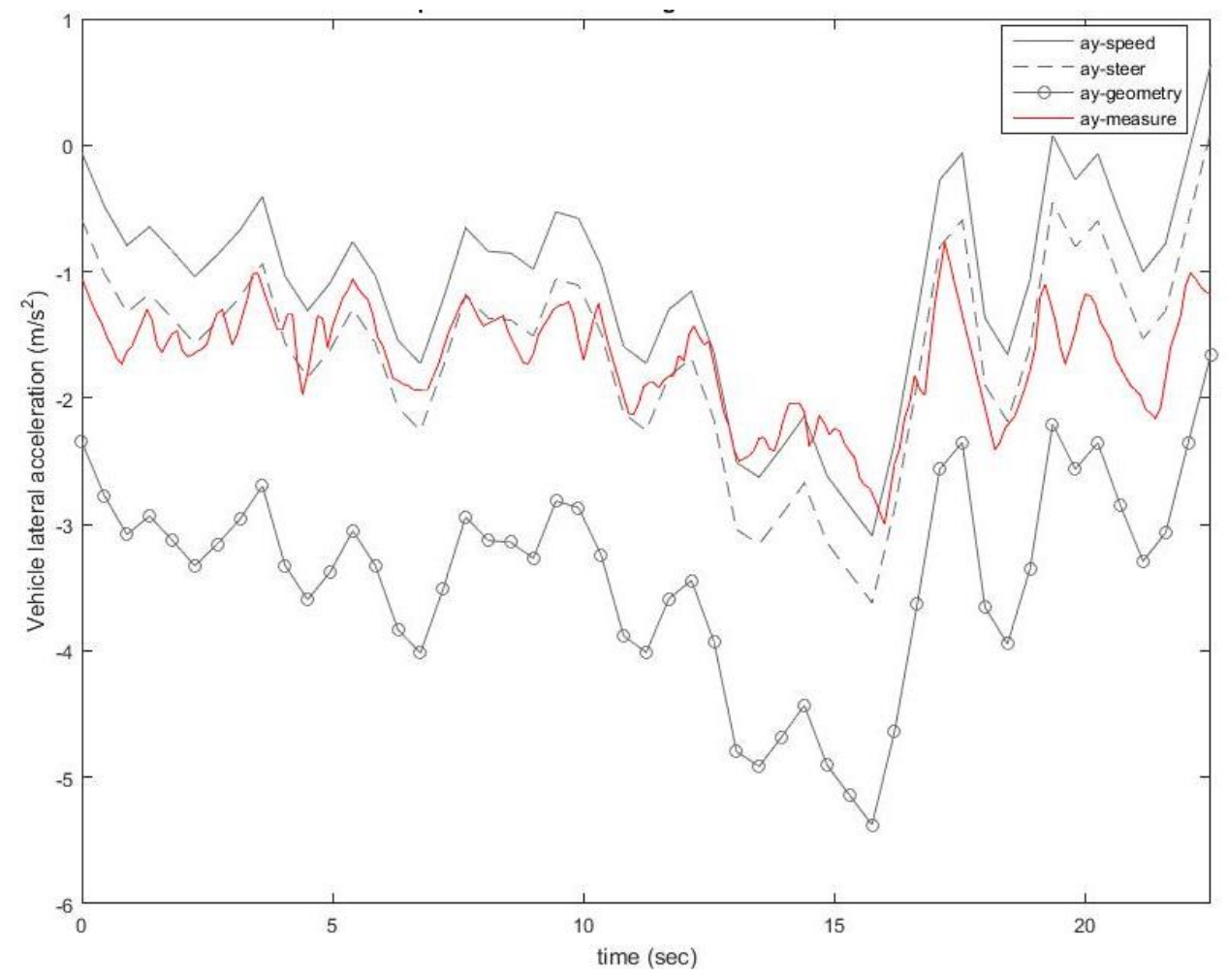

Fig. 12. Acceleration with time for one single turn around the circular track.

\section{Conclusion}

An investigation of the dynamics of narrow track tilting vehicles has been introduced. As a matter of fact, a tilting vehicle model can be constructed under two independent systems, the tilt model and lateral acceleration model. The lateral acceleration can be described by a simplified 'bicycle' model while the tilting speed can be represented by a basic inverted pendulum system. The control strategy utilises the estimated lateral acceleration to compute the required titling angle in order to balance the vehicle during cornering. Therefore, an accurate assessment of this quantity is important to develop the control architecture as well as other safety functions, such as vehicle sliding elimination. The contribution of this article is to propose the modified geometric 'bicycle' model using an uncertain parameter and the simple and effective method for the model parameter identification. It is worth mentioning that, due to limited experimental data available, the current approach is only applicable for offline optimisation under a steady state manoeuvre. As a future work, 
the proof of concept should be implemented on real-time optimisation and validated by the transient vehicle manoeuvres to investigate the vehicle responses under aggressive driver behaviours (rapid steering inputs and vehicle speeds).

\section{List of Abbreviations}

\begin{tabular}{|l|l|}
\hline CLEVER & Compact Low Emission Vehicle for Urban Transport \\
\hline DTC & Direct Tilt Control \\
\hline EU & European Union \\
\hline HILS & Hardware-in-the-Loop Simulation \\
\hline NTV & Narrow Track Vehicle \\
\hline PD controller & Proportional-Derivative controller \\
\hline STC & Steering Tilt Control \\
\hline
\end{tabular}

\section{Acknowledgements}

The research presented in this article was undertaken as part of the Range of Electric Solutions for L Category Vehicles (RESOLVE) Project. Funded through the European Funding for Research and Innovation (Horizon 2020), Grant Number 653511.

\section{References}

Kidane, S., L. Alexander, R. Rajamani, P. Starr and M. Donath (2008), 'A fundamental investigation of tilt control systems for narrow commuter vehicles', Vehicle System Dynamics, 42 (4), 295-322.

Gohl, J., R. Rajamani, L. Alexander and P. Starr (2004), 'Active roll mode control implementation on a narrow tilting vehicle' Vehicle System Dynamics, 42 (5), 347-372.

Furuichi, H., J. Huang, T. Matsuno and T. Fukuda (2012), 'Dynamic model of three wheeled narrow tilting vehicle and optimal tilt controller design', IEEE international symposium on micro-nanomechatronics and human science conference, Nagoya, 4-7 November, 2012.

Amati, N., A. Festini, L. Pelizza and A. Tonoli (2011), 'Dynamic modelling and experimental validation of three wheeled tilting vehicles', Vehicle System Dynamics, 49 (4), 889-914. 
Besselink, I. (2006), 'Vehicle Dynamics analysis using SimMechanics and TNO Delft-Tyre', IAC The Mathworks International Automotive Conference, Stuttgart, 16-17 May, 2006.

Maakaroun, S., Ph. Chevrel, M. Gautier and W. Khalil (2011), 'Modeling and simulation of a two wheeled vehicle with suspensions by using robotic formalism', 18th IFAC World Congress, Milano, 28 August-02 September 2011.

Berote, J. (2010), 'Dynamics and control of a tilting three wheeled vehicle', PhD thesis, University of Bath.

Ryu, J. (2004), 'State and parameter estimation for vehicle dynamics control using GPS', PhD thesis, Stanford University.

Gerdes, J. and Y-H Judy Hsu, A Feel for the Road: A Method to Estimate Tire Parameters Using Steering Torque, http://ddl.stanford.edu/sites/default/files/AVEC2006paper_v3.pdf, accessed 20 July 2016.

Lundquist, C. and T.B. Schön, 'Recursive identification of cornering stiffness parameters for an enhanced single track model', 15th IFAC Symposium on System Identification, Saint-Malo, 6-8 July, 2009.

So, S. and D. Karnopp (1997), 'Switching strategies for narrow ground vehicles with dual mode automatic tilt control' International Journal of Vehicle Design, $18-(5)$, 518-32.

Lu, X., K. Guo, D. Lu and Y.L. Wang (2006), 'Effect of tire camber on vehicle dynamic simulation for extreme cornering', Vehicle System Dynamics, 44, (sup1), 39-49.

Pacejka, H. and E. Bakker (1992), 'The magic formula tyre model', Vehicle System Dynamics, 21 (sup001), 1-18.

Akar, M. and J.C. Kalkkuhl (2008). 'An integrated chassis controller for automotive vehicle emulation', Proceedings of the 17th World Congress, Seoul, 6-11 July, 2008.

Poelgeest, A. (2011), 'The dynamics and control of a three-wheeled tilting vehicle', PhD Thesis, University of Bath.

\section{To cite this article:}

Chong, J., Marco, J., \& Greenwood, D. (2016). Modelling and Simulations of a Narrow Track Tilting Vehicle. Exchanges: The Warwick Research Journal, 4(1), 86- 105. Retrieved from:

http://exchanges.warwick.ac.uk/index.php/exchanges/article/view/133 